\title{
RIGIDITY RESULTS FOR SUBMANIFOLDS WITH PARALLEL MEAN CURVATURE VECTOR IN THE DE SITTER SPACE
}

\author{
A. BRASIL JR \\ Departamento de Matemática-UFC \\ e-mail:aldir@mat.ufc.br \\ URL: http://www.mat.ufc.br \\ ROSA M. B. CHAVES \\ Departamento de Matemática-IMEUSP \\ e-mail:rosab@ime.usp..br \\ URL: http://www.ime.usp.br \\ and A. G. COLARES \\ Departamento de Matemática-UFC \\ e-mail:colares@mat.ufc.br \\ URL: http://www.mat.ufc.br
}

(Received 11 November, 2004; revised 11 November, 2005)

\begin{abstract}
In this work we consider a complete spacelike submanifold $M^{n}$ immersed in the De Sitter space $S_{p}^{n+p}(1)$ with parallel mean curvature vector. We use a Simons type inequality to obtain some rigidity results characterizing umbilical submanifolds and hyperbolic cylinders in $S_{p}^{n+p}(1)$.
\end{abstract}

2000 Mathematics Subject Classification. 53C42, 53A10.

1. Introduction. Let $Q_{p}^{n+p}(c)$ be an $(n+p)$-dimensional connected semiRiemannian manifold of index $p$ and of constant curvature $c$, which is called an indefinite space form of index $p$. If $c=1$, we call it the De Sitter space of index $p$ and denote it by $S_{p}^{n+p}(1)$. Let $M^{n}$ be an $n$-dimensional Riemannian manifold and $\psi: M^{n} \rightarrow S_{p}^{n+p}(1)$ be an immersion. The immersion is said to be spacelike if the induced metric on $M^{n}$ from the metric of $S_{p}^{n+p}(1)$ is positive definite. In 1977 Goddard [7] conjectured that the only complete constant mean curvature spacelike hypersurfaces in the De Sitter space were the umbilical ones. In 1987 Akutagawa [1] proved the Goddard conjecture when $H^{2}<1$ if $n=2$ and $H^{2}<4(n-1) / n^{2}$, if $n>2$. He also showed that when $n=2$, for any constant $H^{2}>c^{2}$ there exists a non-umbilical surface of mean curvature $H$ in the De Sitter space $S_{1}^{3}(c)$ of constant curvature $c>0$. (This result was generalized by Cheng in 1991 [3] to general submanifolds in the De Sitter space.) One year later S. Montiel [12] solved Goddard's problem in the compact case in $S_{1}^{n+1}$ without restriction over the range of $H$. He also gave examples of non-umbilical complete spacelike hypersurfaces in $S_{1}^{n+1}$ with constant mean curvature $H$ satisfying $H^{2} \geq 4(n-1) / n^{2}$, including the so-called hyperbolic cylinders. In [13], Montiel proved that the only complete spacelike hypersurface in $S_{1}^{n+1}$ with constant mean curvature $H=2 \sqrt{n-1} / n$ with more than one topological end is a hyperbolic cylinder. This result was extended by Li [11] in 1997. He proved that a complete spacelike submanifold 
with parallel mean curvature vector with more than one topological end is a hyperbolic cylinder. In 1991, Ki, Kim, Nakagawa [9] obtained an upper bound for the norm of the second fundamental form of the hypersurface in terms of the (constant) mean curvature of the given submanifold and the ambient curvature of the space form and showed that the upper bound is realized by the hyperbolic cylinder. X. Liu ([10]) characterized, in 2001, the complete spacelike submanifolds $M^{n}$ with parallel mean curvature vector satisfying $H^{2}=4(n-1) c / n^{2}(c>0)$ in the De Sitter space $S_{p}^{n+p}(c)$. He showed that $M^{n}$ is totally umbilical, or $M^{n}$ is the hyperbolic cylinder in $S_{p}^{n+p}(c)$ or $M^{n}$ has unbounded volume and positive Ricci curvature.

Recently, Brasil, Colares and Palmas obtained in [2] an interesting gap theorem. More precisely, they considered an immersion $\psi: M^{n} \rightarrow S_{1}^{n+1}(1)$ with mean curvature vector $h$, mean curvature $H=|h|$ and the traceless tensor $\Phi=A-H I$, where $I$ stands for the identity operator. First they used a Simons type inequality for the tensor $\Phi$ and applied that formula to get some results characterizing umbilical hypersurfaces and hyperbolic cylinders in $S_{1}^{n+1}(1)$. In order to state their result let us first introduce totally umbilical hypersurfaces and also hyperbolic cylinders in $S_{1}^{n+1}(1)$.

The totally umbilical hypersurfaces are given by the intersection of $S_{1}^{n+1}$ and affine hyperplanes

$$
P^{n}=\left\{x \in S_{1}^{n+1} ;\langle x, a\rangle=\tau\right\} \subset L^{n+2},
$$

where $a \in L^{n+2},|a|^{2}=\sigma=1,0,-1$ and $\tau^{2}>\sigma$. If $\sigma=1, M^{n}$ is isometric to a hyperbolic space $H^{n}$, with sectional curvature $k=-\frac{1}{\tau^{2}-1}$ and mean curvature $H=$ $\frac{\tau}{\tau^{2}-1}$. If $\sigma=0, M^{n}$ is isometric to $R^{n}$, with sectional curvature $k=0$ and mean curvature $H=1$. If $\sigma=-1, M^{n}$ is isometric to the sphere $S^{n}$, with sectional curvature $k=\frac{1}{\tau^{2}-1}$ and mean curvature $H=-\frac{\tau^{2}}{\tau^{2}-1}$.

Let us denote by $H^{1} \times S^{n-1}$ the hyperbolic cylinders $H^{1}(\sinh r) \times S^{n-1}(\cosh r)$. For more details we refer to $\mathrm{Ki}, \mathrm{Kim}$ and Nakagawa [9]. It is possible to show that these hypersurfaces have one principal curvature equal to coth $r$ and $(n-1)$ principal curvatures equal to $\tanh r$. Thus the mean curvature is given by $n H=\operatorname{coth} r+$ $(n-1) \tanh r$ and $|\Phi|^{2}=\frac{(n-1)}{n}(\operatorname{coth} r-\tanh r)^{2}$.

Now we are ready to state the mentioned result due to Brasil, Colares and Palmas. See [2, Theorem 1.2, p. 849].

THEOREM 1.1. Let $M^{n}, n \geq 3$ be a complete spacelike hypersurface in $S_{1}^{n+1}$ with constant mean curvature $H>0$. Then $\sup |\Phi|^{2}<\infty$ and either

(a) $|\Phi| \equiv 0$ and $M^{n}$ is totally umbilical; or

(b) $B_{H}^{-} \leq \sqrt{\sup |\Phi|^{2}} \leq B_{H}^{+}$, where $B_{H}^{-} \leq B_{H}^{+}$are the roots of the polynomial

$$
P_{H}(x)=x^{2}-\frac{n(n-2)}{\sqrt{n(n-1)}} H x+n\left(1-H^{2}\right) .
$$

In this work we generalize the result above for higher codimensions and apply it to characterize umbilical submanifolds and hyperbolic cylinders in $S_{p}^{n+p}(1)$.

Take an immersion $\psi: M^{n} \rightarrow S_{p}^{n+p}(1)$ and choose a suitable pseudo-orthonormal frame field $\left\{e_{1}, e_{2}, \ldots, e_{n+1}, e_{n+2}, \ldots, e_{n+p}\right\}$ adapted to the immersion $\psi$ and its associated coframe $\left\{\omega_{1}, \omega_{2}, \ldots, \omega_{n+1}, \omega_{n+2}, \ldots, \omega_{n+p}\right\}$. Now recall the symmetric 
traceless tensor introduced by Cheng and Yau in [5], given by

$$
\Phi=\sum_{i, j, \alpha} \Phi_{i j}^{\alpha} \omega_{i} \otimes \omega_{j} \otimes e_{\alpha},
$$

where $i, j=1,2, \ldots, n, \alpha=n+1, n+2, \ldots, n+p, \Phi_{i j}^{\alpha}=h_{i j}^{\alpha}-\frac{1}{n} \operatorname{tr} H^{\alpha} I, \quad H^{\alpha}=\left(h_{i j}^{\alpha}\right)$ and $h_{i j}^{\alpha}$ are the coeficients of the second fundamental form in the direction $e_{\alpha}$.

Our main results can be stated as follows.

THEOREM 1.2. Let $M^{n}(n \geq 3)$ be a complete spacelike submanifold in the De Sitter space $S_{p}^{n+p}(1)$ with parallel mean curvature vector. Then either

(a) $H^{2}<\frac{4(n-1)}{n^{2}}$ and $M^{n}$ is totally umbilical or

(b) $H^{2} \geq \frac{4(n-1)}{n^{2}}$ and $\max \left\{0, \alpha^{-}(n, H, p)\right\} \leq \sup |\Phi| \leq \alpha^{+}(n, H, p)$,

where $\alpha^{-}(n, H, p)$ and $\alpha^{+}(n, H, p)$ are the real roots of the quadratic polynomial

$$
P_{H}(x)=\frac{x^{2}}{p}-\frac{n(n-2)}{\sqrt{n(n-1)}} H x+n\left(1-H^{2}\right) .
$$

Moreover if the equality holds and $M^{n}$ is not totally umbilical then $p=1$.

The result above lets us reduce codimension and characterize umbilical hypersurfaces and hyperbolic cylinders in $S_{1}^{n+1}(1) \subset S_{p}^{n+p}(1)$.

Corollary 1.3. Let $M^{n}(n \geq 3)$ be a complete spacelike submanifold in $S_{p}^{n+p}(1)$ with parallel mean curvature vector and $H^{2} \geq \frac{4(n-1)}{n^{2}}$. If $\sup |\Phi| \leq \max \left\{0, \alpha^{-}(n, H, p)\right\}$ or $\sup |\Phi| \geq \alpha^{+}(n, H, p)$, then either

(a) $M^{n}$ is totally umbilical or

(b) $\sup |\Phi|=\alpha^{-}(n, H, p)>0$ or $\sup |\Phi|=\alpha^{+}(n, H, p)$.

Furthermore, if $\sup |\Phi|=\alpha^{-}(n, H, p)>0$ and this supremum is attained on $M^{n}$, then $M^{n}$ is isometric to a hyperbolic cylinder $H^{1} \times S^{n-1}$.

COROllary 1.4. Let $M^{n}(n \geq 3)$ be a complete spacelike submanifold in $S_{p}^{n+p}(1)$ with parallel mean curvature vector and $H^{2} \geq \frac{4(n-1)}{n^{2}}$. If $M^{n}$ has constant scalar curvature and $\sup |\Phi| \leq \max \left\{0, \alpha^{-}(n, H, p)\right\}$ or $\sup |\Phi| \geq \alpha^{+}(n, H, p)$, then either $M^{n}$ is totally umbilical or $M^{n}$ is isometric to a hyperbolic cylinder $H^{1} \times S^{n-1}$.

2. Preliminaries. Throughout this section we shall introduce some basic facts and notation that will appear in this paper. Now let $M^{n}$ be a complete spacelike submanifold immersed in the De Sitter space $S_{p}^{n+p}(1)$. Choose a pseudo-orthonormal frame field $\left\{e_{1}, e_{2}, \ldots, e_{n+p}\right\}$ in $S_{p}^{n+p}(1)$ such that, at each point of $M^{n},\left\{e_{1}, e_{2}, \ldots, e_{n}\right\}$ is an orthonormal frame that spans the tangent space of $M^{n}$. We use the following standard convention of the range of indices:

$$
1 \leq A, B, C, \ldots, \leq n+p, 1 \leq i, j, k, \ldots, \leq n, n+1 \leq \alpha, \beta, \gamma, \ldots, \leq n+p .
$$

Let $\left\{\omega_{1}, \omega_{2}, \ldots \omega_{n+p}\right\}$ be its dual frame field so that the semi-Riemannian metric of $S_{p}^{n+p}(1)$ is given by $d s^{2}=\sum_{i} \omega_{i}^{2}-\sum_{\alpha} \omega_{\alpha}^{2}=\sum_{A} \epsilon_{A} \omega_{A}^{2}, \epsilon_{i}=1$ and $\epsilon_{\alpha}=-1$.

Then the structure equations of $S_{p}^{n+p}(1)$ are given by

$$
d \omega_{A}=-\sum_{B} \omega_{A B} \wedge \omega_{B}, \omega_{A B}+\omega_{B A}=0,
$$




$$
\begin{gathered}
d \omega_{A B}=-\sum_{C} \epsilon_{C} \omega_{A C} \wedge \omega_{C B}-\frac{1}{2} \sum_{C, D} \epsilon_{C} \epsilon_{D} K_{A B C D} \omega_{C} \wedge \omega_{D}, \\
K_{A B C D}=\epsilon_{A} \epsilon_{B}\left(\delta_{A C} \delta_{B D}-\delta_{A D} \delta_{B C}\right) .
\end{gathered}
$$

Next, we restrict those forms to $M^{n}$. First of all $\omega_{\alpha}=0$. Then

$$
0=d \omega_{\alpha}=-\sum_{i} \omega_{\alpha i} \wedge \omega_{i}
$$

and, from Cartan's Lemma, we can write

$$
\omega_{\alpha i}=\sum_{j} h_{i j}^{\alpha} \omega_{j}, \quad h_{i j}^{\alpha}=h_{j i}^{\alpha} .
$$

We call $B=\sum_{\alpha, i, j} h_{i j}^{\alpha} \omega_{i} \omega_{j} e_{\alpha}$,

$$
h=\frac{1}{n} \sum_{i, \alpha} h_{i i}^{\alpha} e_{\alpha}
$$

and

$$
H=|h|=\frac{1}{n} \sqrt{\sum_{\alpha}\left(\sum_{i} h_{i i}^{\alpha}\right)^{2}}
$$

respectively, the second fundamental form, the mean curvature vector and the mean curvature of $M^{n}$.

The structure equations of $M^{n}$ are given by

$$
\begin{gathered}
d \omega_{i}=-\sum_{j} \omega_{i j} \wedge \omega_{j}, \quad \omega_{i j}+\omega_{j i}=0, \\
d \omega_{i j}=-\sum_{k} \omega_{i k} \wedge \omega_{k j}-\frac{1}{2} \sum_{k, l} R_{i j k l} \omega_{k} \wedge \omega_{l} .
\end{gathered}
$$

If $R_{i j k l}$ and $R_{\alpha \beta k l}$ stand for the tensor of curvature and normal curvature, then the Gauss equation can be read as follows:

$$
R_{i j k l}=\delta_{i k} \delta_{j l}-\delta_{i l} \delta_{j k}-\sum_{\alpha}\left(h_{i k}^{\alpha} h_{j l}^{\alpha}-h_{i l}^{\alpha} h_{j k}^{\alpha}\right) .
$$

The components of the Ricci curvature tensor Ric and the scalar curvature $R$ are given, respectively, by

$$
\begin{gathered}
R_{j k}=(n-1) \delta_{j k}-\sum_{\alpha, i}\left(h_{i i}^{\alpha} h_{j k}^{\alpha}+h_{i k}^{\alpha} h_{j i}^{\alpha}\right), \\
R=n(n-1)-n^{2} H^{2}+S,
\end{gathered}
$$

where $S=\sum_{\alpha, i, j}\left(h_{i j}^{\alpha}\right)^{2}$ is the squared norm of $B$.

We state also the structure equations of the normal bundle of $M^{n}$

$$
d \omega_{\alpha}=-\sum_{\beta} \omega_{\alpha \beta} \wedge \omega_{\beta}, \omega_{\alpha \beta}+\omega_{\beta \alpha}=0,
$$




$$
\begin{gathered}
d \omega_{\alpha \beta}=-\sum_{\gamma} \omega_{\alpha \gamma} \wedge \omega_{\gamma \beta}-\frac{1}{2} \sum_{i, j} R_{\alpha \beta i j} \omega_{i} \wedge \omega_{j}, \\
R_{\alpha \beta i j}=-\sum_{l}\left(h_{i l}^{\alpha} h_{j l}^{\beta}-h_{j l}^{\alpha} h_{i l}^{\beta}\right) .
\end{gathered}
$$

The covariant derivatives of $h_{i j k}^{\alpha}$ of $h_{i j}^{\alpha}$ satisfy

$$
\sum_{k} h_{i j k}^{\alpha} \omega_{k}=d h_{i j}^{\alpha}-\sum_{k} h_{k i}^{\alpha} \omega_{k j}-\sum_{k} h_{j k}^{\alpha} \omega_{k i}-\sum_{\beta} h_{i j}^{\beta} \omega_{\beta \alpha} .
$$

Then, by the exterior differentiation of (2.5), we obtain the Codazzi equation

$$
h_{i j k}^{\alpha}=h_{i k j}^{\alpha}=h_{j i k}^{\alpha} .
$$

Similarly, we have the second covariant derivatives $h_{i j k l}^{\alpha}$ of $h_{i j}^{\alpha}$ so that

$$
\sum_{l} h_{i j k l} \omega_{l}=d h_{i j k}^{\alpha}-\sum_{l} h_{l j k}^{\alpha} \omega_{l i}-\sum_{l} h_{i l k}^{\alpha} \omega_{l j}-\sum_{l} h_{i j l}^{\alpha} \omega_{l k}-\sum_{\beta} h_{i j k}^{\beta} \omega_{\beta \alpha},
$$

and it is easy to get the following Ricci formula

$$
h_{i j k l}^{\alpha}-h_{i j l k}^{\alpha}=-\sum_{m} h_{i m}^{\alpha} R_{m j k l}-\sum_{m} h_{j m}^{\alpha} R_{m i k l}-\sum_{\beta} h_{i j}^{\beta} R_{\alpha \beta k l} .
$$

The Laplacian $\Delta h_{i j}^{\alpha}$ of $h_{i j}^{\alpha}$ is defined by $\Delta h_{i j}^{\alpha}=\sum_{k} h_{i j k k}^{\alpha}$. From (2.17) we have

$$
\Delta h_{i j}^{\alpha}=\sum_{k} h_{k k i j}^{\alpha}-\sum_{k, m} h_{k m}^{\alpha} R_{m i j k}-\sum_{k, m} h_{m i}^{\alpha} R_{m k j k}-\sum_{\beta, k} h_{k i}^{\beta} R_{\alpha \beta j k} .
$$

We recall that $M^{n}$ is a submanifold with parallel mean curvature vector if $\nabla^{\perp} h=0$ on $M^{n}$, where $\nabla^{\perp}$ is the normal connection of $M^{n}$ in $S_{p}^{n+p}(1)$. From now on we assume that $M^{n}$ is a complete spacelike submanifold in $S_{p}^{n+p}(1)$ with parallel mean curvature vector, which implies that the mean curvature $H$ is constant. If $H=0, M^{n}$ is maximal and from [8] we know that $M^{n}$ is totally geodesic. If $H \neq 0$, we choose $e_{n+1}=\frac{h}{H}$. Thus

$$
\operatorname{tr} H^{n+1}=n H, \quad \operatorname{tr} H^{\alpha}=0, \quad \alpha \geq n+2,
$$

where $H^{\alpha}$ denotes the matrix $\left(h_{i j}^{\alpha}\right)$,

$$
\sum_{k} h_{k k i}^{\alpha}=0, \quad \forall i, \alpha
$$

and

$$
\omega_{\alpha, n+1}=0, \quad H^{\alpha} H^{n+1}=H^{n+1} H^{\alpha}, \quad R_{n+1 \alpha i j}=0 .
$$

By replacing (2.10), (2.15), (2.20) and (2.21) in (2.18), we get

$$
\begin{aligned}
\Delta h_{i j}^{n+1}= & n h_{i j}^{n+1}-n H \delta_{i j}+\sum_{\beta, k, m} h_{k m}^{n+1} h_{m k}^{\beta} h_{i j}^{\beta}-2 \sum_{\beta, k, m} h_{k m}^{n+1} h_{m j}^{\beta} h_{i k}^{\beta}+\sum_{\beta, k, m} h_{m i}^{n+1} h_{m k}^{\beta} h_{k j}^{\beta} \\
& -n H \sum_{m} h_{m i}^{n+1} h_{m j}^{n+1}+\sum_{\beta, k, m} h_{j m}^{n+1} h_{m k}^{\beta} h_{k i}^{\beta},
\end{aligned}
$$


and for $\alpha \geq n+2$, we have

$$
\begin{aligned}
\Delta h_{i j}^{\alpha}= & n h_{i j}^{\alpha}+\sum_{\beta, k, m} h_{k m}^{\alpha} h_{m k}^{\beta} h_{i j}^{\beta}-2 \sum_{\beta, k, m} h_{k m}^{\alpha} h_{m j}^{\beta} h_{i k}^{\beta} \\
& +\sum_{\beta, k, m} h_{m i}^{\alpha} h_{m k}^{\beta} h_{k j}^{\beta}-n H \sum_{m} h_{m i}^{\alpha} h_{m j}^{n+1}+\sum_{\beta, k, m} h_{j m}^{\alpha} h_{m k}^{\beta} h_{k i}^{\beta} .
\end{aligned}
$$

Since

$$
\frac{1}{2} \Delta S=\sum_{\alpha, i, j, k}\left(h_{i j k}^{\alpha}\right)^{2}=\frac{1}{2} \sum_{\alpha, i, j}\left(\Delta h_{i j}^{\alpha}\right)^{2}=\sum_{\alpha, i, j} h_{i j}^{\alpha} \Delta h_{i j}^{\alpha}+\sum_{\alpha, i, j, k}\left(h_{i j k}^{\alpha}\right)^{2},
$$

by using (2.21), (2.22) and (2.23) it is straightforward to verify that

$$
\begin{aligned}
\frac{1}{2} \Delta S= & \sum_{\alpha, i, j, k}\left(h_{i j k}^{\alpha}\right)^{2}+n S-n^{2} H^{2}-n H \sum_{\alpha} \operatorname{tr}\left(H^{n+1}\left(H^{\alpha}\right)^{2}\right) \\
& +\sum_{\alpha, \beta}\left[\operatorname{tr}\left(H^{\alpha} H^{\beta}\right)\right]^{2}+\sum_{\alpha, \beta} N\left(H^{\alpha} H^{\beta}-H^{\beta} H^{\alpha}\right),
\end{aligned}
$$

where $N(A)=\operatorname{tr}\left(A A^{t}\right)$, for each matrix $A=\left(a_{i j}\right)$.

In the next section we shall need also the following lemma due to Omori [15] and Yau [19].

Lemma 2.1. Let $M^{n}$ be an n-dimensional complete Riemannian manifold whose Ricci curvature is bounded from below. Let $f$ be a $C^{2}$-function bounded from below on $M^{n}$. Then for each $\epsilon>0$ there exists a point $p_{\epsilon} \in M^{n}$ such that $|\nabla f|\left(p_{\epsilon}\right)<\epsilon, \Delta f\left(p_{\epsilon}\right)>$ $-\epsilon$ and $\inf f \leq f\left(p_{\epsilon}\right)<\inf f+\epsilon$.

We also need the following algebraic lemma, due to Santos. See [17, p. 407].

Lemma 2.2. Let $A, B: R^{n} \rightarrow R^{n}$ be symmetric linear maps such that $A B-B A=0$ and $\operatorname{tr} A=\operatorname{tr} B=0$. Then

$$
-\frac{n-2}{\sqrt{n(n-1)}}\left(\operatorname{tr} A^{2}\right)\left(\operatorname{tr} B^{2}\right)^{\frac{1}{2}} \leq \operatorname{tr} A^{2} B \leq \frac{n-2}{\sqrt{n(n-1)}}\left(\operatorname{tr} A^{2}\right)\left(\operatorname{tr} B^{2}\right)^{\frac{1}{2}}
$$

and the equality holds on the right hand (resp. left hand) side if and only if $n-1$ of the eigenvalues $x_{i}$ of $A$ and the corresponding eigenvalues $y_{i}$ of B satisfy

$$
\begin{gathered}
\left|x_{i}\right|=\frac{\left(\operatorname{tr} A^{2}\right)^{\frac{1}{2}}}{\sqrt{n(n-1)}}, \quad x_{i} x_{j} \geq 0, \\
y_{i}=\frac{\left(\operatorname{tr} B^{2}\right)^{\frac{1}{2}}}{\sqrt{n(n-1)}}\left(\operatorname{resp} \cdot y_{i}=-\frac{\left(\operatorname{tr} B^{2}\right)^{\frac{1}{2}}}{\sqrt{n(n-1)}}\right) .
\end{gathered}
$$

3. Proofs of the rigidity results. In this section we are going to use a version of Simons' inequality for submanifolds in the De Sitter space $S_{p}^{n+p}(1)$ to prove the first rigidity result. That inequality was recently obtained by Chaves and Sousa in [4] and the authors kindly let us present their proof here, in order to clarify our own proof. H. Li, [11] already obtained some related formulae, estimating separately $|\Phi|^{2}$ in the 
directions $e_{n+1}$ and the remaining ones. Chaves and Sousa's result can be stated as follows.

THEOREM 3.1 (A Simons type inequality). Let $M^{n}$ be a complete spacelike submanifold in the De Sitter space $S_{p}^{n+p}(1)$ with parallel mean curvature vector. Then the following inequality holds:

$$
\frac{1}{2} \Delta|\Phi|^{2} \geq|\Phi|^{2}\left(\frac{|\Phi|^{2}}{p}-\frac{n(n-2)}{\sqrt{n(n-1)}} H|\Phi|+n\left(1-H^{2}\right)\right) .
$$

Proof. Since $\nabla^{\perp} h=0$, the mean curvature $H$ is constant. If $H \equiv 0, M^{n}$ is called maximal. In this case $\sum_{i} h_{i i}^{\alpha}=0$ for all $\alpha$ which, together with (2.24) imply that

$$
\begin{aligned}
\frac{1}{2} \Delta|\Phi|^{2}= & \frac{1}{2} \Delta S=\sum_{\alpha, i, j, k}\left(h_{i j k}^{\alpha}\right)^{2}+n S+\sum_{\alpha, \beta}\left(\operatorname{tr} H^{\alpha} H^{\beta}\right)^{2} \\
& +\sum_{\alpha, \beta} N\left(H^{\beta} H^{\beta}-H^{\beta} H^{\beta}\right) \geq n S+\sum_{\alpha} N^{2}\left(H^{\alpha}\right) \\
= & n|\Phi|^{2}+\sum_{\alpha} N^{2}\left(H^{\alpha}\right) \geq|\Phi|^{2}\left(\frac{|\Phi|^{2}}{p}+n\right) .
\end{aligned}
$$

If $H \neq 0$, we choose the adapted orthonormal frame such that $e_{n+1}=\frac{h}{H}$. Since $e_{n+1}$ is a parallel direction, it is easy to verify that

$$
\begin{aligned}
& \Phi_{i j}^{n+1}=H_{i j}^{n+1}-H \delta_{i j}, \quad \Phi_{i j}^{\alpha}=H_{i j}^{\alpha}, \quad \alpha \geq n+2, \\
& \Phi^{n+1}=H^{n+1}-H I, \quad \Phi^{\alpha}=H^{\alpha}, \quad \alpha \geq n+2, \\
& N\left(\Phi^{n+1}\right)=\operatorname{tr}\left(\Phi^{n+1}\right)^{2}=\operatorname{tr}\left(H^{n+1}\right)^{2}-n H^{2},
\end{aligned}
$$

where $\Phi^{\alpha}$ and $H^{\alpha}$ denote, respectively, the matrices $\left(\Phi_{i j}^{\alpha}\right)$ and $\left(h_{i j}^{\alpha}\right)$. Moreover

$$
\operatorname{tr}\left(H^{n+1}\right)^{3}=\operatorname{tr}\left(\Phi^{n+1}\right)^{3}+3 H \operatorname{tr}\left(\Phi^{n+1}\right)^{2}+n H^{3} .
$$

By replacing (3.3) and (3.4) in (2.24) we get

$$
\begin{aligned}
\frac{1}{2} \Delta|\Phi|^{2}= & \sum_{\alpha, i, j, k}\left(\Phi_{i j k}^{\alpha}\right)^{2}+n\left(1-H^{2}\right)|\Phi|^{2}-n H \sum_{\alpha} \operatorname{tr}\left(\Phi^{n+1}\left(\Phi^{\alpha}\right)^{2}\right) \\
& +\sum_{\alpha, \beta}\left(\operatorname{tr} \Phi^{\alpha} \Phi^{\beta}\right)^{2}+\sum_{\alpha, \beta} N\left(\Phi^{\alpha} \Phi^{\beta}-\Phi^{\beta} \Phi^{\alpha}\right) .
\end{aligned}
$$

By applying Lemma 2.2 to $\Phi^{\alpha}$ and $\Phi^{n+1}$ we get

$$
\left|\operatorname{tr}\left(\Phi^{n+1}\left(\Phi^{\alpha}\right)^{2}\right)\right| \leq \frac{n-2}{\sqrt{n(n-1)}} \sqrt{N\left(\Phi^{n+1}\right)} N\left(\Phi^{\alpha}\right) .
$$

By definition

$$
\begin{gathered}
N\left(\Phi^{\alpha}\right)=\sum_{i, j}\left(\Phi_{i j}^{\alpha}\right)^{2}, \\
|\Phi|^{2}=\sum_{\alpha} N\left(\Phi^{\alpha}\right)=\sum_{\alpha, i, j}\left(\Phi_{i j}^{\alpha}\right)^{2}=S-n H^{2} .
\end{gathered}
$$


Now taking the summand for all $\alpha \neq n+1$ on the right side of inequality (3.6) and using (3.7) we get

$$
\begin{aligned}
\sum_{\alpha} \operatorname{tr}\left(\Phi^{n+1}\left(\Phi^{\alpha}\right)^{2}\right) & \leq \frac{n-2}{\sqrt{n(n-1)}} \sqrt{N\left(\Phi^{n+1}\right)} \sum_{\alpha} N\left(\Phi^{\alpha}\right) \\
& \leq \frac{n-2}{\sqrt{n(n-1)}}|\Phi|^{3} .
\end{aligned}
$$

Using the Cauchy-Schwarz inequality, it is easy to prove that

$$
|\Phi|^{4} \leq p \sum_{\alpha} N^{2}\left(\Phi^{\alpha}\right) \leq p \sum_{\alpha, \beta}\left(\operatorname{tr} \Phi^{\alpha} \Phi^{\beta}\right)^{2} .
$$

It follows from (3.5), (3.8) and (3.9) that formula (3.1) holds. This completes the proof of the Simons type inequality.

Now we are ready to prove our main theorem.

Proof of Theorem 1.2. If $H^{2}<\frac{4(n-1)}{n^{2}}$ it follows from Cheng's Theorem (see [3]) that $M^{n}$ is totally umbilical. Let us suppose $H^{2} \geq \frac{4(n-1)}{n^{2}}$. In this case it is easy to see that $P_{H}(x)$ has two real roots $\alpha^{-}(n, H, p) \leq \alpha^{+}(n, H, p)$. Consider the positive smooth function $F$ on $M^{n}$ defined by $F=\frac{1}{\sqrt{1+|\Phi|^{2}}}$. By a simple calculation we obtain

$$
|\nabla F|^{2}=\frac{\left.\left.|\nabla| \Phi\right|^{2}\right|^{2}}{4\left(1+|\Phi|^{2}\right)^{3}} \text { and } F \Delta F=\frac{-\Delta|\Phi|^{2}}{2\left(1+|\Phi|^{2}\right)^{2}}+3|\nabla F|^{2} .
$$

Now, from (3.1) and (3.10) we obtain

$$
F \Delta F \leq \frac{-|\Phi|^{2}}{2\left(1+|\Phi|^{2}\right)^{2}}\left(\frac{|\Phi|^{2}}{p}-\frac{n(n-2)}{\sqrt{n(n-1)}} H|\Phi|+n\left(1-H^{2}\right)\right)+3|\nabla F|^{2} .
$$

Assume that the second fundamental form of $M^{n}$ with respect to $e_{n+1}$ has been diagonalized so that the eigenvalues are $\lambda_{i}^{n+1}$. From (2.11) we have

$\operatorname{Ric}\left(e_{i}\right) \geq(n-1)-n H h_{i i}^{n+1}+\sum_{k}\left(h_{i k}^{n+1}\right)^{2}=\left(\lambda_{i}^{n+1}-\frac{n H}{2}\right)^{2}+(n-1)-\frac{n^{2} H^{2}}{4}$.

Hence the Ricci curvature of $M^{n}$ is bounded from below. Since $M^{n}$ is spacelike and $F>0$, we can apply Lemma 2.1 to the function $F$. Thus we can obtain a sequence of points $\left\{p_{k}\right\}$ in $M^{n}$ such that

$$
\begin{gathered}
\lim _{k \rightarrow \infty} F\left(p_{k}\right)=\inf (F) ; \\
\lim _{k \rightarrow \infty}|\Phi|^{2}\left(p_{k}\right)=\sup |\Phi|^{2}=(\sup |\Phi|)^{2} ; \\
\lim _{k \rightarrow \infty}\left|\nabla F\left(p_{k}\right)\right|=0 ; \quad \lim _{k \rightarrow \infty} \inf \Delta F\left(p_{k}\right) \geq 0 .
\end{gathered}
$$

By replacing (3.13) in (3.11) we get

$$
\frac{\sup |\Phi|^{2}}{\left(1+\sup |\Phi|^{2}\right)^{2}}\left(\frac{\sup |\Phi|^{2}}{p}-\frac{n(n-2)}{\sqrt{n(n-1)}} H \sup |\Phi|+n\left(1-H^{2}\right)\right) \leq 0 .
$$


Inequality (3.14) shows that $\sup |\Phi|<\infty$. Moreover it implies that $\sup |\Phi|=0$ or $\max \left\{0, \alpha^{-}(n, H, p)\right\} \leq \sup |\Phi| \leq \alpha^{+}(n, H, p)$.

Let us assume that $\sup |\Phi|=\alpha^{-}(n, H, p)>0$ or $\sup |\Phi|=\alpha^{+}(n, H, p)$. In particular these assumptions imply that $M^{n}$ is not totally umbilical. Assuming further that $p \geq 2$ we shall derive a contradiction.

Since $|\Phi|^{2}=\sum_{\alpha} N\left(\Phi^{\alpha}\right)$ is a smooth function bounded from above on $M^{n}$, we can apply again Lemma 2.1 to the function $|\Phi|^{2}$. Therefore, taking subsequences if necessary, we can obtain a sequence $\left(p_{k}\right)$ in $M^{n}$ such that

$$
\begin{gathered}
\lim _{k \rightarrow \infty}|\Phi|^{2}\left(p_{k}\right)=\sup |\Phi|^{2}=(\sup |\Phi|)^{2}, \\
\lim _{k \rightarrow \infty} N\left(\Phi^{\alpha}\right)\left(p_{k}\right)=C_{\alpha}^{2}, \quad \alpha=n+1, \ldots, n+p, \\
\left.\lim _{k \rightarrow \infty}|\nabla| \Phi\right|^{2}\left(p_{k}\right) \mid=0, \\
\lim _{k \rightarrow \infty} \sup \left(\Delta|\Phi|^{2}\right)\left(p_{k}\right) \leq 0,
\end{gathered}
$$

where $C_{\alpha}$ are constants.

Remember that in order to prove inequality (3.1) we used inequalities (3.8) and (3.9). Then equality holds in (3.1) if and only if it holds in formulae (3.8) and (3.9). By applying inequalities (3.1), (3.8) and (3.9) at $\left(p_{k}\right)$, approaching the limit and using (3.15) we get

$$
\begin{gathered}
0 \geq \frac{1}{2} \lim _{k \rightarrow \infty} \sup \left(\Delta|\Phi|^{2}\right)\left(p_{k}\right) \\
\geq \sup |\Phi|^{2}\left(\frac{\sup |\Phi|^{2}}{p}-\frac{n(n-2)}{\sqrt{n(n-1)}} H \sup |\Phi|+n\left(1-H^{2}\right)\right) \\
C_{n+1} \sup |\Phi|^{2} \leq \sup |\Phi|^{3} \\
\left(\sup |\Phi|^{2}\right)^{2} \leq p \sum_{\alpha} C_{\alpha}^{4} .
\end{gathered}
$$

As we are assuming that $\sup |\Phi|=\alpha^{-}(n, H, p)>0$ or $\sup |\Phi|=\alpha^{+}(n, H, p)$, both sides of inequality (3.16) are actually equal to zero. As before, equality holds in (3.16) if and only if it holds in (3.17) and (3.18). More precisely, (3.17) and (3.18) can be rewritten as the following equalities

$$
\begin{aligned}
C_{n+1}(\sup |\Phi|)^{2}= & C_{n+1} \sup |\Phi|^{2}=\sup |\Phi|^{3}=(\sup |\Phi|)^{3}, \\
& \left(\sup |\Phi|^{2}\right)^{2}=p \sum_{\alpha} C_{\alpha}^{4} .
\end{aligned}
$$

Since $M^{n}$ is not totally umbilical, (3.19) implies $C_{n+1}=\sup |\Phi|>0$ and so the second formula of (3.19) yields $(p-1) C_{n+1}^{4}+p \sum_{\alpha=n+2}^{n+p} C_{\alpha}^{4}=0$. Hence, if $p \geq 2$, as all the constants $C_{\alpha}$ are non-negative, we infer that $0=C_{n+1}=\sup |\Phi|$ and $M^{n}$ is totally umbilical. This contradiction shows that $p=1$ and finishes our proof.

Proof of Corollary 1.3. From Theorem 1.2 we conclude that $p=1$ and the proof then follows from Proposition 1.1 of [2]. Observe that the hypothesis $H<1$ in the cited proposition just appeared to ensure that $\alpha^{-}(n, H, 1)$ was positive. (See formula (12) of [2].) But we already have this assured by assumption. 
Proof of Corollary 1.4. The hypothesis and Corollary 1.3 imply that either $M^{n}$ is totally umbilical or $\sup |\Phi|=\alpha^{-}(n, H, p)$ or $\sup |\Phi|=\alpha^{+}(n, H, p)$. If $\sup |\Phi|=$ $\alpha^{+}(n, H, p)$ from Theorem 2 of [9] we conclude that $M^{n}$ is isometric to a hyperbolic cylinder. If $\sup |\Phi|=\alpha^{-}(n, H, p)$, as we are assuming constant scalar curvature, by (2.12) we get that $S$ is constant and then $|\Phi|$ is also constant. Consequently, sup $|\Phi|$ is attained on $M^{n}$ and the last assertion of Corollary 1.3 implies again that $M^{n}$ is isometric to a hyperbolic cylinder.

ACKNOwledgement. The authors would like to thank the referee for many valuable suggestions that really improved the paper.

\section{REFERENCES}

1. K. Akutagawa, On spacelike hypersurfaces with constant mean curvature in the De Sitter space, Math. Z. 196 (1987), 13-19.

2. A. Brasil, G. Colares and O. Palmas, Complete spacelike hypersurfaces with constant mean curvature in the De Sitter space: a gap theorem, Illinois J. Math. 47 (2003), 847-866.

3. Q. M. Cheng, Complete space-like submanifolds in De Sitter space with parallel mean curvature vector, Math. Z. 206 (1991), 333-339.

4. R. M. B. Chaves and L. A. M. Sousa Jr, A Simons type formula for submanifolds with parallel mean curvature vector in the De Sitter space and applications, preprint, 2005.

5. S. Y. Cheng and S. T. Yau, Hypersurfaces with constant scalar curvature, Math. Ann. 225 (1977), 195-204.

6. M. Dajczer and K. Nomizu, On flat surfaces in $S_{1}^{3}$ and $H_{1}^{3}$, in Manifolds and Lie groups (Birkhauser, 1981), 71-88.

7. A. J. Goddard, Some remarks on the existence of spacelike hypersurfaces of constant mean curvature, Math. Proc. Cambridge Phil. Soc. 82 (1977), 489-495.

8. T. Ishihara, Maximal space-like submanifolds in a pseudo-Riemannian space of constant curvature, Mich. Math. J. 35 (1988), 345-352.

9. U. H. Ki, H. J. Kim and H. Nakagawa, On spacelike hypersurfaces with constant mean curvature of a Lorentz space form, Tokyo J. Math. 14 (1991), 205-216.

10. X. Liu, Complete space-like submanifolds in de Sitter space, Acta Math. Univ. Comenianae LXX(2) (2001), 241-249.

11. H. Li, Complete spacelike submanifolds in de Sitter space with parallel mean curvature vector satisfying $H^{2}=\frac{4(n-1)}{n^{2}}$, Ann. Global Anal. Geom. 15 (1997), 335-345.

12. S. Montiel, An integral inequality for compact spacelike hypersurfaces in the de Sitter space and applications to the case of constant mean curvature, Indiana Univ. Math. J. 37 (1988), 909-917.

13. S. Montiel, A characterization of hyperbolic cylinders in the de Sitter space, Tôhoku Math. J. 48 (1996), 23-31.

14. V. Oliker, A priori estimates of the principal curvature of spacelike hypersurfaces in the de Sitter space with applications to hypersurfaces in hyperbolic space, Amer. J. Math. 144 (1992), 605-626.

15. H. Omori, Isometric Immersions of Riemannian manifolds, J. Math. Soc. Japan 19 (1967), 205-214.

16. J. Ramanathan, Complete spacelike hypersurfaces of constant mean curvature in the de Sitter space, Indiana University Math. J. 36 (1987), 349-359.

17. W. Santos, Submanifolds with parallel mean curvature vector in spheres, Tôhoku Math. J. 46 (1994), 403-415.

18. R. Schoen and S. T. Yau, Lectures on differential geometry (International Press, Cambridge, 1994).

19. S. T. Yau, Harmonic functions on complete Riemannian manifolds, Comm. Pure Appl. Math. 28 (1975), 201-228. 